\title{
Sensor Saturation in Fourier Multiplexed Imaging
}

\author{
Gordon Wetzstein \\ wetzste1@cs. ubc.ca \\ The University of British Columbia
}

\author{
Ivo Ihrke* \\ ivoihrkeecs.ubc.ca \\ The University of British Columbia \\ $\left(^{*}\right)$ now at Saarland University
}

\author{
Wolfgang Heidrich \\ heidrich@cs.ubc.ca \\ The University of British Columbia
}

\begin{abstract}
Optically multiplexed image acquisition techniques have become increasingly popular for encoding different exposures, color channels, light fields, and other properties of light onto two-dimensional image sensors. Recently, Fourier-based multiplexing and reconstruction approaches have been introduced in order to achieve a superior light transmission of the employed modulators and better signalto-noise characteristics of the reconstructed data.

We show in this paper that Fourier-based reconstruction approaches suffer from severe artifacts in the case of sensor saturation, i.e. when the dynamic range of the scene exceeds the capabilities of the image sensor. We analyze the problem, and propose a novel combined optical light modulation and computational reconstruction method that not only suppresses such artifacts, but also allows us to recover a wider dynamic range than existing image-space multiplexing approaches.
\end{abstract}

\section{Introduction and Related Work}

Photography has evolved as one of the primary means by which we represent and communicate the three-dimensional world around us. Over the past decade, we have seen a push to digital photo-sensors, such as charge coupled device (CCD) and complementary metal-oxidesemiconductor (CMOS) sensors, which have largely replaced traditional film photography.

Digital sensors, however, have a relatively limited dynamic range compared to both the human visual system and photographic film. The recent development of consumer displays that support a high dynamic range [24] has increased the demand for high contrast content beyond the scope of movie theaters.

In order to capture a high dynamic range image or other visual information with standard digital sensors, a variety of multiplexing techniques have been proposed. One of the most popular approaches is to successively capture different exposures of the same scene with a single camera $[9,17$, 23]. Alternatively, multiple aligned image sensors can be employed to simultaneously capture images of a scene that are either differently exposed or filtered $[4,16]$. The latter approach is costly, while the former usually does not allow for acquisition of dynamic environments.

Different optical information can also be encoded into a single photograph. Examples of this approach include high dynamic range imaging [19], photography with color filter arrays (CFAs) [7, 8], and light field acquisition [14, 2, 20, 13]. These techniques can be generalized as Assorted Pixels [18], where each sensor pixel captures an exposure or some other part of the plenoptic function [3]. Micro-lens arrays can be equipped with different apertures to capture high dynamic range light fields [10].

Recently, more sophisticated multiplexing schemes have been explored. Modulators that optically encode light properties in different spatial frequency bands have been proposed for the acquisition of light fields [26, 25] and occluder information [12]. The corresponding reconstruction has usually been performed in the Fourier domain, although a recent analysis [11] shows that a spatial reconstruction is possible. Compared to standard spatial multiplexing techniques, Fourier multiplexing methods allow for a superior light transmission and potentially increased signal-to-noise ratio (SNR) of the demultiplexed signal.

Although the effect of sensor saturation on spatial reconstructions of multiplexed data is well understood, it has so far been ignored for Fourier-based reconstruction methods. We analyze the problem and show that saturation results in severe artifacts for these approaches as the global frequency content is altered by the saturation.

Inspired by the idea of Fourier-based image reconstruction, we present a joint optical light modulation and computational reconstruction approach to boosting the dynamic range of multiplexed photographs. Previously, plausible dynamic range values were estimated from demosaicked images using heuristics $[22,6,15]$ or priors on the color distributions for the case of a single saturated color channel in a photograph [28]. In contrast to this, we develop a numerical optimization method for Fourier-based reconstruction and dynamic range boosting of multiplexed data that precedes the demosaicking step and recovers the raw sensor data.

Restoration of clipped general [1] and ultrasonic [21] signals based on inequality constrained optimization has been successful, but it does not consider the artifacts introduced by reconstructing individual channels from a satu- 
rated multiplexed signal.

Specifically, our contributions are

- An analysis of the effects of sensor saturation for multiplexed image reconstruction in the Fourier domain (Section 2).

- A numerical optimization method for Fourier-based dynamic range boosting of multiplexed data. The approach is introduced for dynamic range multiplexing with neutral density filters (Section 3).

- A prototype that allows novel color filter arrays and other multiplexing masks to be developed and tested in a macroscopic scale (Section 4).

- A novel color filter array that allows our dynamic range boosting technique to be applied to full color photographs (Section 5).

\section{Saturation Analysis in Fourier Space}

The principle of Fourier multiplexed image reconstruction is illustrated in Figure 1. A band-limited optical signal is filtered with a periodic light modulator that is mounted directly on the sensor, such as a color filter array (CFA) or an array of different neutral density (ND) filters. The optical filtering, or multiplication, of incident light and modulator is, according to the convolution theorem, equivalent to a convolution in the Fourier domain. The convolution enables a copy mechanism that allows Fourier multiplexing approaches to directly encode desired visual information in different frequency bands of the image. As shown in Figure 1 (lower right), these can then be cropped in the Fourier transform of the sensor image and individually transformed back to the spatial domain.

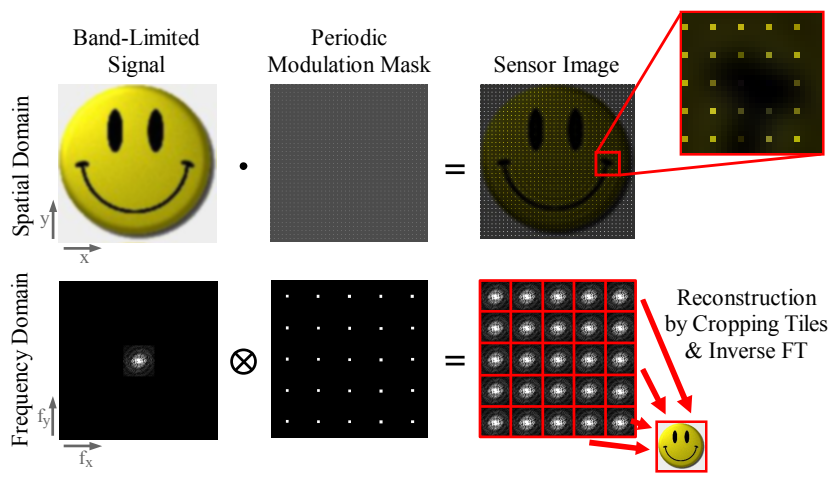

Figure 1. A simulated, band-limited optical signal is filtered with a semi-transparent mask placed directly in front of the image sensor. This multiplication (upper row) corresponds to a convolution in the frequency domain (lower row). Fourier multiplexing exploits the resulting copy mechanism to capture several differently filtered versions of a scene in different frequency bands of a photograph.

Although specialized Fourier multiplexing masks have so far only been used to acquire light fields $[26,25,12]$ it is straightforward to extend this concept to color filter arrays (see Section 5). The potential of Fourier-based reconstruction methods for color demosaicking of raw CFA imagery, even with standard Bayer filters, has only recently been discovered [5].

In order to understand the effects of sensor saturation on the individual Fourier tiles, let us consider a 1D unmodulated, band-limited scene as shown in Figure 2 (left). Due to the band-limited nature of the signal, only low image frequencies are present that occupy a single frequency band (Fig. 2, lower left), while all other frequency bands are empty and reserved for additional information to be optically encoded with a modulator. Sensor saturation, as illustrated in Figure 2 (right), destroys the band-limited nature of the signal by modifying the frequency content of the signal. The frequency bands that were originally reserved for additional data are corrupted with high frequency components.
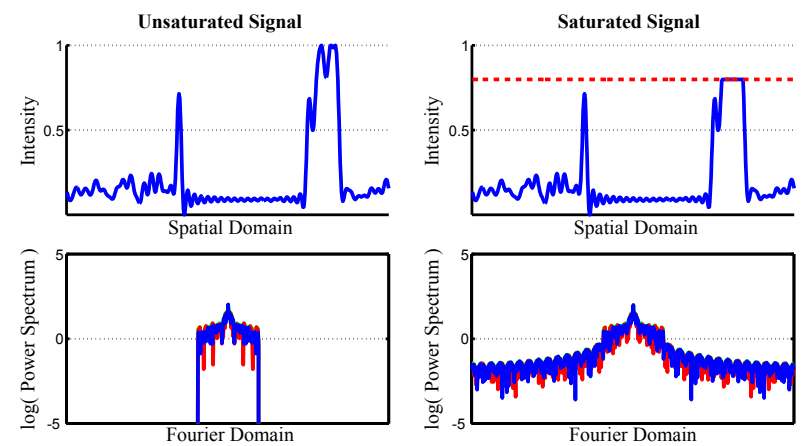

Figure 2. A band-limited signal (upper left), consisting of a single scanline taken from a high dynamic range image, and the same signal clipped at 0.8 maximum intensity level (upper right). The Fourier transform of the original scanline is band-limited (lower left), while the Fourier transform of the clipped version of the same scanline is corrupted by high frequency components.

In order to understand the effect of saturation, let us now consider a simple example scene, shown in Figure 3, where a constant white signal is captured through an attenuation mask consisting of different neutral density (ND) filters. The Fourier transform of the underlying signal is a single value for the DC term. According to the Poisson summation formula, the Fourier transform of the unsaturated periodic sensor image is a series of differently weighted Fourier peaks as seen in Figure $3 \mathrm{~b}$. If the dynamic range of this mask-modulated scene exceeds that of the sensor, some of the ND filters are saturated (c), and the global scale of the Fourier copies is altered (d). In this case only parts of each super-pixel, that is one spatial period of the modulator, are saturated; therefore, we refer to these regions as being partially saturated.

Another case of saturation occurs when the scene regionally exhibits a very high dynamic range. Here, one or more spatially neighboring super-pixels are fully saturated. Due to the spatial structure of the super-pixels being completely removed in such regions, the local information cannot be copied into the high frequency bands of the image. Instead, other frequency bands are corrupted.

In summary, saturation has a significant impact on the 


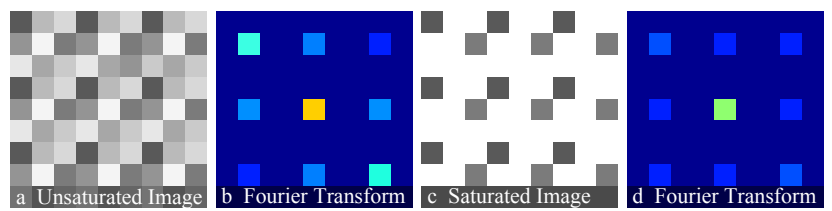

Figure 3 . A constant signal captured through a $3 \times 3$ pattern of neutral density filters (a) and its Fourier transform (b). Partial saturation of this sensor image (c) causes changes in the magnitudes of the signal's Fourier copies (d). Peak magnitudes are color-coded.

performance of Fourier multiplexing techniques. Saturation, if not dealt with properly, can introduce severe artifacts in multiplexed information. In the next section, we demonstrate how to recover lost information and extend the dynamic range of the captured content.

\section{Dynamic Range Optimization}

Based on our previous analysis, we now introduce an optimization approach to recover dynamic range in the saturated regions of a multiplexed image. As seen in Figure 2, even a band-limited signal has contributions in all frequency bands when saturation occurs. Previous approaches $[1,21]$ to restoring the original signal in this case constrain the frequency bands (1D) or tiles (2D) that are not occupied by the unsaturated signal to be zero. Unfortunately, these constrains are infeasible for multiplexed data, because all frequency bands are used by the encoded channels and therefore non-zero.

For the case of multiplexing a monochromatic signal with an array of neutral density filters, we know that the unsaturated multiplexed image encodes differently scaled versions of the same scene in each tile (see Fig. 1). The individual scales are determined by the transmission and layout of the ND filters. In the presence of saturation, however, we have seen that each channel is corrupted. Following the idea of constraining the reconstructed signal, or in our case each individual channel, to be band-limited, we propose to add a zero-constraint to the differences of the properly scaled frequency tiles. This enforces each of the reconstructed channels to be band-limited and directly proportional to all the other channels.

Let us denote each of the $N$ frequency tiles of a 2D sensor image $L(x, y)$ as $T_{i}\left(f_{x}, f_{y}\right)=s_{i} \cdot\left(F_{i}\{L(x, y)\}+\eta_{i}\right)$, where $\eta_{i}$ is the Fourier-transformed sensor noise, and $s_{i}$ is the scale of frequency tile $i$ introduced by the modulation mask. $F_{i}$ projects the full-resolution sensor image into a single frequency tile and can be constructed from the DFT matrix by zeroing all the rows that do not belong to tile $i$. The squared $\ell_{2}$-norm of the tile differences is given as

$$
\varepsilon=\sum_{i=1}^{N-1} \sum_{j=i+1}^{N}\left\|T_{i}\left(f_{x}, f_{y}\right)-T_{j}\left(f_{x}, f_{y}\right)\right\|_{2}^{2} .
$$

For notational simplicity we assume in the following that all tiles have been normalized by dividing through the corresponding factor after capture.
In addition to the prior placed on the channel differences we also need to incorporate the measured observations, which are the unsaturated intensity values of the multiplexed image. For this purpose, we split the maskmodulated sensor image $L$ into a part where the sensor pixels saturate, and a second part where they do not. That is $L=L_{\text {unsat }}+L_{\text {sat }}$, where

$$
\begin{array}{r}
L_{\text {unsat }}(x, y)= \begin{cases}L(x, y) & ; L(x, y)<L_{\max } \\
0 & ; \text { else }\end{cases} \\
L_{\text {sat }}(x, y)= \begin{cases}0 & ; L(x, y)<L_{\max } \\
L_{\max } & ; \text { else }\end{cases}
\end{array}
$$

The challenge is to estimate the parts of the sensor image that are saturated during capture, i.e. to replace $L_{\max }$ in the $L_{\text {sat }}$ image by values satisfying the Fourier domain constraint imposed by our setup. Since the Fourier transform is linear, the additive relationship between $L_{\text {sat }}$ and $L_{\text {unsat }}$ holds for the Fourier representations of the signal components: $F\{L\}=F\left\{L_{\text {unsat }}\right\}+F\left\{L_{\text {sat }}\right\}$. The individual tiles are therefore given as

$$
T_{i}=F_{i}\left\{L_{\text {unsat }}\right\}+F_{i}\left\{L_{\text {sat }}\right\}+\eta_{i} .
$$

The term $F_{i}\left\{L_{\text {unsat }}\right\}$ can readily be computed from the captured image, and represents measured data. $F_{i}\left\{L_{\text {sat }}\right\}$ includes the unknown variables (the non-zero subset of $L_{\text {sat }}$ ) that causes the saturation error in the Fourier domain. Combining Equations 1 and 2 yields

$$
\begin{aligned}
\varepsilon=\sum_{i=1}^{N-1} \sum_{j=i+1}^{N} \| F_{i}\left\{L_{\text {unsat }}\right\}-F_{j}\left\{L_{\text {unsat }}\right\} & \\
& +F_{i}\left\{L_{\text {sat }}\right\}-F_{j}\left\{L_{\text {sat }}\right\}+\eta_{i}+\eta_{j} \|_{2}^{2} .
\end{aligned}
$$

We assume that the sensor noise is independently distributed in the spatial domain and observes a zero-mean Gaussian distribution in the per-pixel image intensities. Thus, $\eta_{i}$ has a uniform power spectrum with a Gaussian characteristic in each Fourier coefficient. This simple noise model allows us to use a quadratic error norm for optimization in Fourier space.

We encode $\varepsilon$ in a linear system of equations, where we optimize the spatial pixel intensities $L_{\text {sat }}$ using an error metric defined in Fourier space. We show a simplified example assuming one copy at the DC peak and one copy of equal scale in a higher frequency band:

$$
\min \left\|\left[\begin{array}{rrr}
1 & -1 & 0 \\
0 & 0 & 0 \\
0 & -1 & 1
\end{array}\right]\left[\begin{array}{l}
F_{1} \\
F_{D C} \\
F_{1}^{*}
\end{array}\right]\left(L_{\text {unsat }}+L_{\text {sat }}\right)\right\|_{2}^{2} .
$$

In matrix notation, Equation 4 becomes $\min \left\|R F\left(L_{\text {unsat }}+L_{\text {sat }}\right)\right\|_{2}^{2}$, with $R$ encoding the relationship between the different Fourier copies, and $F$ 
performing the transformation from the spatial domain into the individual frequency tiles. Because we are minimizing differences, matrix $R$ does not have full rank. We compensate for this by adding a regularizer $S=\Delta F^{-1} F_{D C}$ favoring smooth spatial reconstructions of the DC Fourier tile. Note that the implementation of this idea is complicated by the fact that the optimization procedure recovers the mask-modulated image which contains high frequencies everywhere. Therefore, $F_{D C}$ extracts the DC Fourier tile, while $F^{-1}$ transforms it back into the spatial domain in tile resolution. The Laplacian $\Delta$ then enforces spatial smoothness. The choice of this regularizer is justified by the assumption of a band-limited signal:

$$
\min _{L_{\mathrm{sat}}}\left\|R F\left(L_{\mathrm{unsat}}+L_{\mathrm{sat}}\right)\right\|_{2}^{2}+\alpha\left\|S\left(L_{\mathrm{unsat}}+L_{\mathrm{sat}}\right)\right\|_{2}^{2} .
$$

Differentiating Equation 5 with respect to $L_{\text {sat }}$ and equating the gradient with zero, we obtain a least squares description of our error measure:

$$
\begin{aligned}
& \left(F^{*} R^{*} R F+\alpha S^{*} S\right) L_{\mathrm{sat}}= \\
& \quad-\left(F^{*} R^{*} R F+\alpha S^{*} S\right) L_{\text {unsat }}
\end{aligned}
$$

Note that the right hand side of this system is constant and represents our image space measurements.

Our combined error metric and regularizer boost the reconstructed intensity values beyond the limits that are imposed by the optical ND filters. The smoothness prior imposed by $S$ effectively applies similar inequality constrains as proposed in $[1,21]$, because it penalizes possible solutions with infeasible pixel values in the saturated regions as these would most certainly be non-smooth. Unlike simple inequalities, our approach also ensures smooth transitions between saturated and unsaturated image regions.

Equation 6 describes the final optimization process that we use to estimate lost dynamic range. The equation can be solved using any linear solver. In our work, we employ conjugate gradients for least-squares (CGLS), combined with image-space operations, which allows us to represent the system without explicitly forming the matrix. The result of the optimization process is a restored mask-modulated image where the saturated image regions have been replaced by optimized ND-filter values. A high dynamic range photograph can then be obtained by the procedure described in [19].

\section{Experimental Validation}

Prototype. To validate this optimization procedure on real data, we built a digital camera from a flatbed scanner [27]. This camera is easy to construct, provides a very high-resolution, and is large-scale, which makes it easy to use simple transparencies as masks. Rather than focusing the camera directly on the sensor and applying color filters outside the camera as proposed by Wang and Heidrich [27], we attach our modulation masks directly onto the glass plate, where the incoming light as well as the underlying sensor elements are focused. A holographic diffuser, which allows the incident light to form an image, is also mounted over the filter on the scanner glass plate as seen in Figure 4. It simultaneously serves as a band-limiter for the light incident on the sensor.

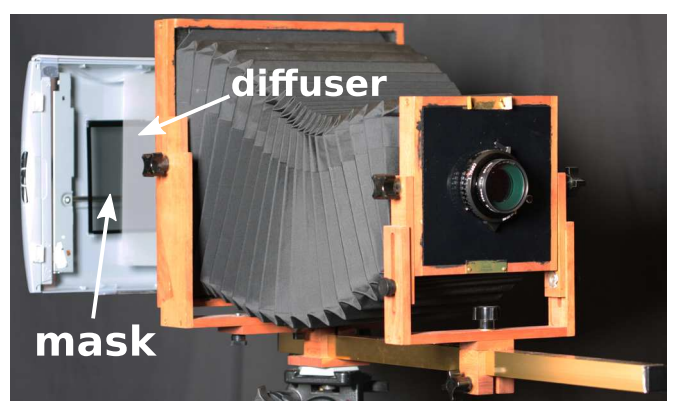

Figure 4. Our prototype is a large format high-resolution scanner camera. The filters are mounted under a diffuser on the scanner's glass plate.

Our masks are high resolution RGB digital images, exposed onto photographic film using light-valve technology (LVT). Color transparencies with a resolution of up to 2032 dpi and a high contrast can be ordered at professional print service providers such as Bowhaus (www.bowhaus.com). We use 4"x5" transparencies and perform scans with 2400 dpi.

Due to slight mis-registrations (rotation and shift) of the filter in front of the sensor in our prototype camera, as well as dust and scanner sensor artifacts, the point spread functions (PSFs) of the filter tiles in the Fourier domain do not exactly correspond to the filter specification before the print. In order to calibrate for these effects, we estimate the PSFs of the individual filter tiles in the Fourier transform of a calibration image.

Optimization Results. An example scene containing saturated regions is shown in Figure 5. Two magnifications of the multiplexed image captured with our scan camera prototype $(2628 \times 1671 \mathrm{px})$ are shown on the upper left (a, top). Three of the Fourier tiles are individually transformed back into the spatial domain and presented above the Fourier transform of the image (b). One of them is also enlarged in Figure 6. Note that they are differently affected by saturation and sensor noise, as well as scanner artifacts. Figure 5 (c) shows the tone-mapped result of our HDR reconstruction. In order to compute it, we performed our optimization on a grid of $3 \times 3$ tiles in the Fourier domain, each with a resolution of $876 \times 557$ pixels. The data was in the range of $0-1$ and our optimization algorithm converged in about 750 iterations to a residual of $10^{-6}$. The large number of iterations can be explained by the very high noise level of our camera prototype. The dynamic range of the captured scene is extended by a factor of 1.58 in this case. Note that this factor is obtained after the tiles have already been di- 


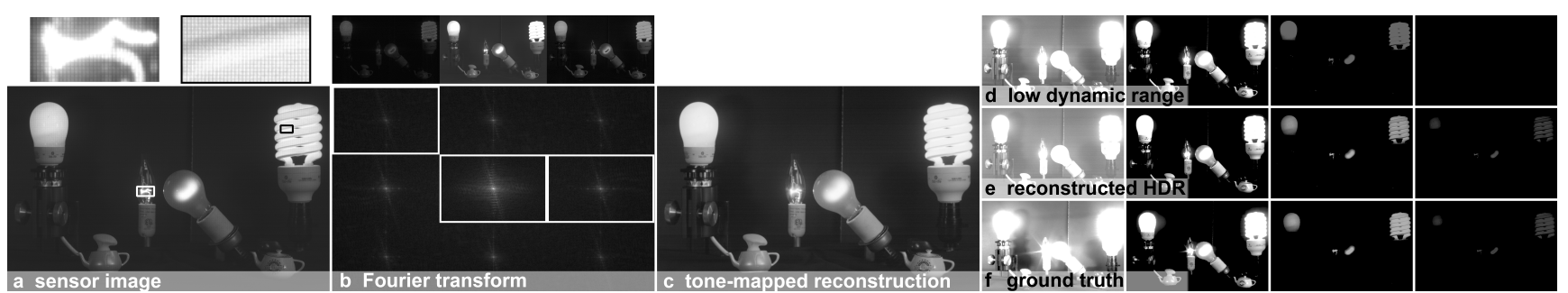

Figure 5. A mask-modulated LDR image captured with a prototype scan camera (a). The pattern introduced by the mask and saturated regions are enlarged on the upper left. The Fourier transform of the captured image (b) contains nine copies, of which three (b, top) are individually transformed into the spatial domain and reveal saturation artifacts (see Figure 6 for enlargement). The tone-mapped result (c) does not contain the mask pattern or Fourier copies. The right part shows a linearly mapped exposure sequence of an unmodulated LDR image (d) and our reconstruction (e) compared to ground truth images (f).

vided by their relative intensity scale factors $s_{i}$, as described in Section 3. Therefore, the factor of 1.58 is an additional improvement on top of the one that would be obtained by using the same neutral density filter array in combination with Assorted Pixel spatial reconstruction [19]. The total gain in dynamic range compared to an unmodulated image is 1.58 times the contrast of the used filters.

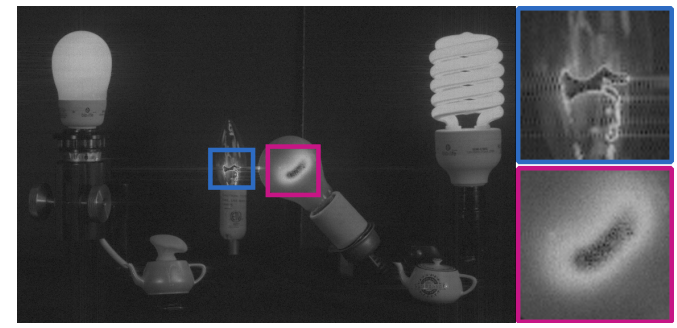

Figure 6. Sensor saturation yields ringing and other artifacts in Fourier multiplexed data. This image is the spatial version of one of the higher frequency tiles in Figure 5.

For validation, we compare our result to a high dynamic range ground truth image that was generated by combining 12 exposures of an SLR camera located next to the scan camera. As seen in the multi-exposure sequence on the right side of Figure 5 (e), the dynamic range can be faithfully recovered. Note in particular the structure recovered in the cold fluorescent light bulb. The depicted LDR image (d) was photographed using our scan camera without the attenuation mask, and lacks details in bright image regions. The SLR image is shown at the bottom (f).

Another example scene is presented in Figure 7. Here, we also show results of our optimization for saturation in dark regions (highlighted in green), as well as in bright image parts (red highlights). The magnifications in the lower row illustrate how saturation $(\mathrm{c}, \mathrm{d})$ is recovered $(\mathrm{e}, \mathrm{f})$ using our approach. Due to the noise floor in the sensor image, our approach can, in this case, not successfully push the intensity values below the blacklevel in dark image regions. Although saturated pixels in the close-ups (c,d) should be entirely flat, errors are introduced by camera noise and the calibration image of the mask which distort the final reconstruction slightly.

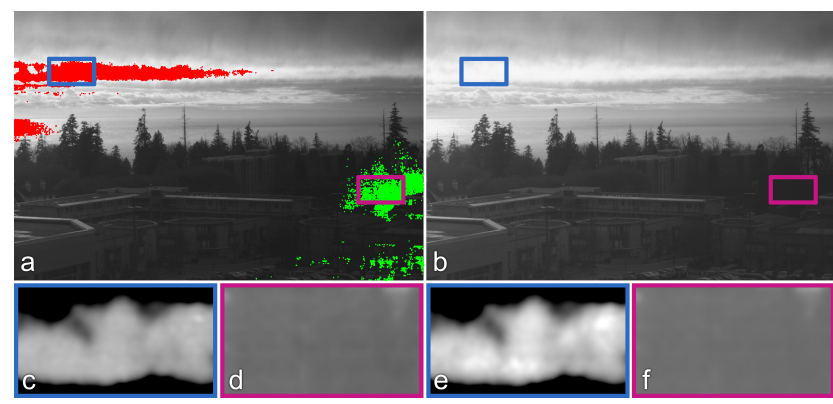

Figure 7. Outdoor scene captured with our scan camera prototype. The sensor image (a) has saturated parts indicated in red (bright) and green (dark); (b) is the reconstructed HDR image. The magnified parts show linearly mapped intensities before (c,d) and after $(\mathrm{e}, \mathrm{f})$ reconstruction for bright and dark saturation respectively.

Comparison to Spatial Reconstruction. We show comparisons of our image reconstruction and the Assorted Pixels approach [19] for a 1D and a 2D scene in Figures 8 and 9 respectively. For the former case we simulated a 1D sensor image by multiplying a repetitive array of neutral density (ND) filters with a test signal taken from a real HDR image and saturating it at $8 \%$ of the dynamic range of the scene. The Assorted Pixels approach [19] performs the reconstruction by dividing by the ND mask values followed by a bicubic interpolation to estimate the saturated pixels (blue line in Figure 8, right). Alternatively, we can apply our Fourierbased reconstruction approach to get a much better estimate of the original function (cyan-colored line in Figure 8).

The 2D comparison presented in Figure 9 shows three different exposures of an HDR image (left column), reconstructions of a simulated sensor image with the Assorted Pixels approach (center column), as well as our method (right column). The sensor image was saturated at $7 \%$ of the dynamic range of the scene. The mask was in this case a repeating pattern of $2 \times 2 \mathrm{ND}$ filters with transmission values of $1,0.5,0.25$, and 0.125 . As expected, dividing by the mask and performing a bi-cubic interpolation can only recover a maximum image intensity that is defined by the ND filter with the lowest light transmission. Our approach can boost the recovered intensity beyond that limit. 


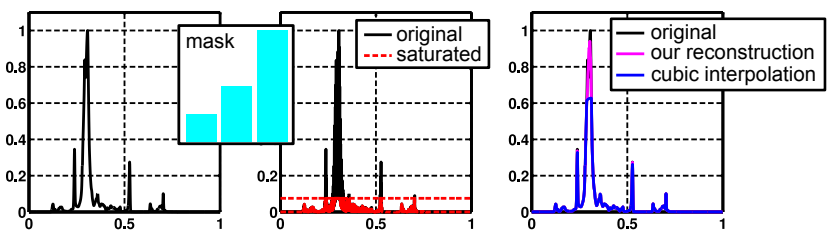

Figure 8. A band-limited 1D signal (left) is modulated with an attenuation mask pattern (one of the repeating tiles shown in inset), and captured by a simulated sensor with a limited dynamic range (red dotted line, center). Our reconstruction (right, magenta) performs better than previously developed interpolation methods (right, blue).

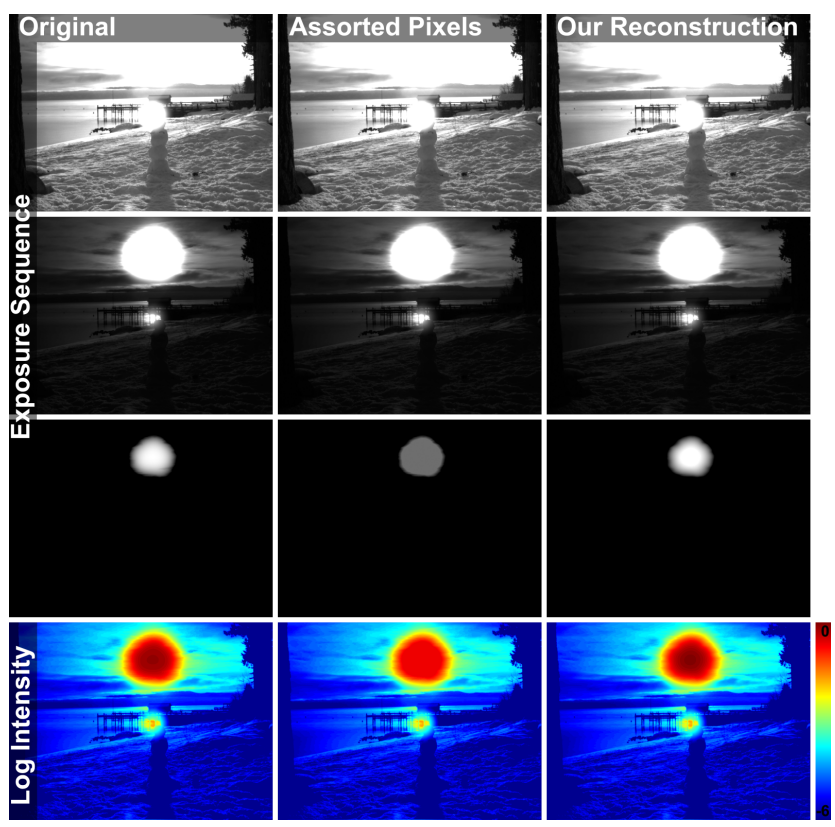

Figure 9. A 2D comparison of previously proposed interpolation and our reconstruction for a repeating $2 \times 2$ pattern of neutral density filters. The simulated sensor image is saturated at $7 \%$ of the dynamic range of the scene. Three different exposures of the HDR images are shown in rows 1-3 and color coded intensities are visualized in the lower row.

Limitations. For very large areas of saturation, we expect our method to eventually produce unsatisfying images, because the regularization term results in an over-smoothing of such regions. In order to test the performance of our algorithm under extreme situations, we photographed a scene that exhibits a very high dynamic range with a number of different aperture settings using our scanner camera prototype as seen in Figure 10. As the size of fully saturated regions grows, an unregularized solution of Equation 6 would become less stable, thus the regularization term counteracts this by filling in smooth image data. The gain in dynamic range is highest for an aperture of 32 (c), because the saturated region is not too large, and the lost dynamic range of the original image is higher than that of the smaller apertures. The gain, however, gets lower for aperture 22 (d), as the equation system becomes more ill-conditioned; the regularization term starts to dominate and over-smooth the so- lution. The effect is even stronger for aperture 11 (e), where we stopped the optimization after 1000 iterations without convergence. We expect a smooth and plausible solution with more iterations, this example just demonstrates slower convergence behavior for larger saturated regions and the effect of over-smoothed solutions. Artifacts in the captured images with aperture settings 22 (d) and 11 (e) are caused by sensor blooming in our prototype camera.

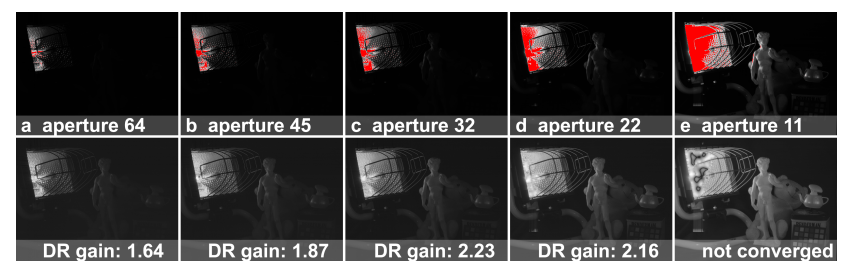

Figure 10. A set of images captured with different camera apertures. For very large fully saturated regions, the optimization oversmoothes the solution. Saturated sensor images are shown in the upper row, tonemapped HDR reconstructions in the lower. The right case did not yet converge for the maximum of 1000 iterations.

\section{Combined Color \& HDR Multiplexing}

So far we have only considered the grayscale case. In this section, we show how to incorporate our reconstruction approach into a Fourier multiplexed capture of color images. This is practically useful, as we introduce a novel color filter array that, in combination with our dynamic range boosting technique, allows us to simultaneously capture RGB imagery and a high dynamic range.

Our color filter array is inspired by a recent analysis of standard Bayer CFAs in the Fourier domain. As discovered by Alleyson et al. [5], a raw sensor image captured through a Bayer pattern inherently contains four differently filtered copies of the image in the Fourier domain. Specifically, these copies are one luminance tile $(R+2 G+B)$, two similar chrominance tiles $(R-B)$, and a fourth tile $(R-2 G+B)$. Instead of redundantly encoding the same chrominance tile twice $(R-B)$, we propose a CFA design that contains two different chrominance tiles $(R-G)$ and $(B-G)$ as well as two differently scaled luminance tiles $(R+2 G+B)$ in the Fourier transform. Our CFA, just like a Bayer pattern, comprises a repeating pattern of colored super-pixels with a resolution of $2 \times 2$ pixels. Rather than sampling each color channel directly, the color distribution for each of our CFA's super-pixels in the spatial domain is

$R=\left(\begin{array}{cc}0.5 & 0.25 \\ 0 & 0.25\end{array}\right), G=\left(\begin{array}{cc}0.5 & 0.25 \\ 0.25 & 1\end{array}\right), B=\left(\begin{array}{cc}0.5 & 0 \\ 0.25 & 0.25\end{array}\right)$

Figure 11 shows a raw sensor image captured with our scanner camera prototype through the proposed color filter array. The photograph contains saturation (a); the spatial pattern of our CFA and a corresponding region of the sensor image are magnified (a, upper left). We illustrate the 

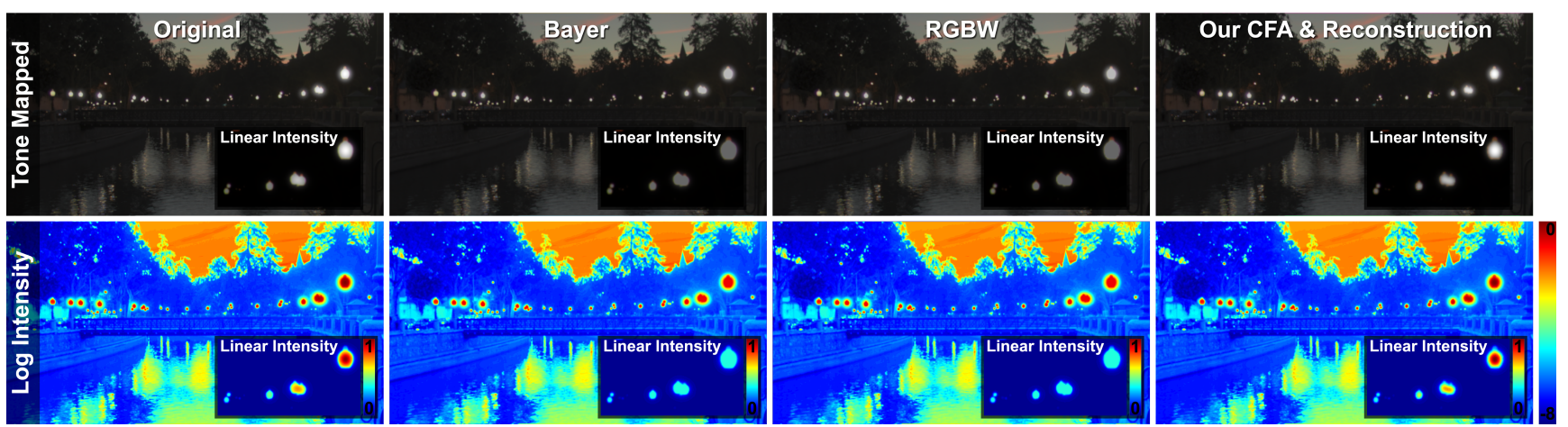

Figure 12. A comparison of imaging through a standard Bayer color filter array (center left column), an RGBW CFA with the same light transmission (center right column), and our CFA with the proposed reconstruction (right column). A sensor image was simulated for all three cases with a dynamic range of $4 \%$ of that of the photographed scene. The upper row shows the tone mapped original image and the three reconstructions with linearized magnifications. The lower row shows color coded logarithmic intensities and linear intensities in the magnifications.
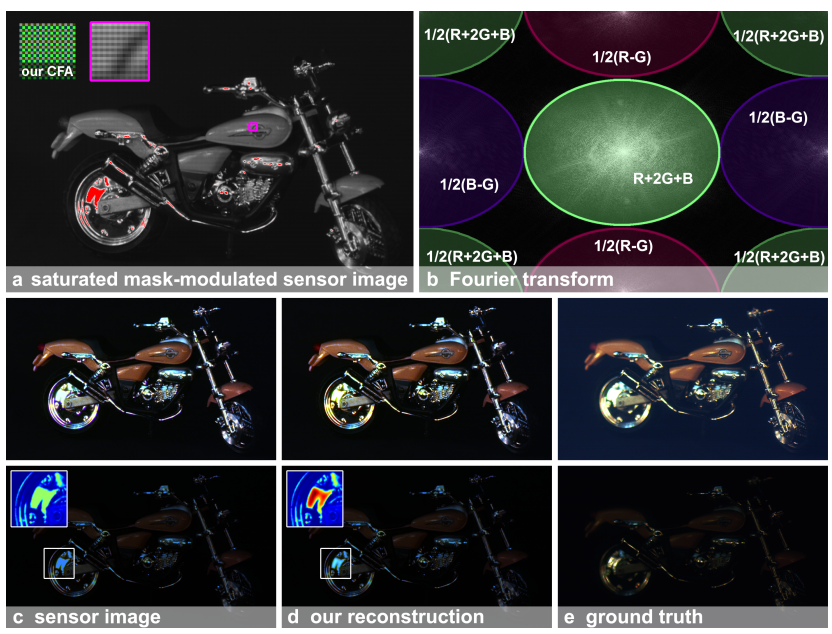

Figure 11. A sensor image captured with our prototype through a novel CFA with saturated regions in red (a). The mask creates two differently scaled luma and two chroma copies in the Fourier transform (b). The dynamic range of the captured image can be significantly extended using our optimization approach as seen in the recolored magnifications in the lower images.

chrominance and differently scaled luminance tiles in the Fourier domain (b).

Our optimization can, in this case, be applied to the two different luminance tiles. This implies that no color information can be recovered in saturated regions, as the dynamic range is only boosted in the luminance channel. The lower part of Figure 11 shows two exposures for different reconstructions of the example scene. The left column (c) is directly reconstructed from the saturated sensor image. It therefore represents an image that is comparable to one captured through a Bayer pattern. Our reconstruction (column d) can significantly increase the dynamic range and reconstruct saturated image regions. The right column (e) shows an HDR image assembled from photographs of the scene without our mask, but with pure red, green, and blue filters, and 3 different aperture settings each. A gain factor of 1.9 in dynamic range could be achieved for our reconstruction, as compared to the standard reconstruction from the sensor image. The high noise level of our camera prototype results in a different black level for the ground truth and mask modulated images. Color differences can be explained by imperfect PSF calibration.

A synthetic result is shown in Figure 12. For this example, the original HDR image (left column) was modulated by a Bayer pattern and reconstructed with standard color demosaicking (center left column). Additionally, we simulated a sensor image by applying an RGBW CFA (center right column) with the same mean light transmission as a Bayer pattern and our CFA. The RGBW CFA consists of one red, green, and blue filter each, and an additional white ND filter. A sensor image with our CFA was simulated and saturated at the same intensity as the Bayer and the RGBW sensor images. The result of our reconstruction is shown in the right column of Figure 12. We can see that the dynamic range of the reconstruction can be significantly boosted by our approach.

Many alternative CFA designs are possible, the one presented in this section is just one example for a design that is compliant with our optimization approach. The average light transmission of our CFA is similar to that of a Bayer pattern and the same band-limitation requirements apply, as both CFAs consist of repeating $2 \times 2$ super-pixels.

\section{Discussion}

In summary, we have presented an analysis of saturation artifacts for Fourier-based reconstruction approaches of multiplexed imaging. Based on this analysis, we have proposed an optimization framework that uses optically encoded information in the Fourier transform to facilitate the suppression of such artifacts, as well as an expansion of the dynamic range. Rather than trying to estimate plausible intensity values in saturated regions of demosaicked 
images using heuristics $[22,6,15]$, we use a joint optical light modulation and computational reconstruction approach to optically encode data that guides the customized post-processing algorithms. We have shown limitations of the proposed reconstruction, and have presented an approach for applying our framework to boosting the luminance of Fourier multiplexed color images.

In the future we wish to experiment with alternative color masks, and test our optimization in the context of further optical Fourier multiplexing applications, such as light fields. An interesting avenue of future research is the possibility of integrating our approach into a spatial reconstruction of Fourier multiplexed data [11]. In this case our optimization could be performed as a pre-processing step before the actual image reconstruction, e.g. color demosaicking in the case of multiplexed color channels.

Acknowledgments This work was supported by Dolby under Wolfgang Heidrich's Dolby Research Chair. Gordon Wetzstein was supported by a Walter C. Koerner Fellowship and a UBC Four Year Fellowship and Ivo Ihrke by the Alexander von Humboldt Foundation and the DFG Cluster of Excellence 'Multimodal Computing and Interaction'. We would like to thank the anonymous reviewers for their valuable comments.

\section{References}

[1] J. S. Abel and J. O. Smith. Restoring a Clipped Signal. In Int. Conf. on Acoustics, Speech, and Signal Processing (ICASSP), pages 1745-1748. IEEE, 1991. 1, 3, 4

[2] E. Adelson and J. Wang. Single Lens Stereo with a Plenoptic Camera. IEEE Trans. PAMI, 14(2):99-106, 1992. 1

[3] E. H. Adelson and J. R. Bergen. The Plenoptic Function and the Elements of Early Vision. In Computational Models of Visual Processing, pages 3-20. MIT Press, 1991. 1

[4] M. Aggarwal and N. Ahuja. Split Aperture Imaging for High Dynamic Range. IJCV, 58(1):7-17, 2004. 1

[5] D. Alleyson, S. Süsstrunk, and J. Hérault. Linear Demosaicing inspired by the Human Visual System. IEEE Trans. Im. Proc., 14(4):439-449, 2005. 2, 6

[6] F. Banterle, P. Ledda, K. Debattista, and A. Chalmers. Inverse Tone Mapping. In Proc. GRAPHITE, pages 349-356, 2006. 1,8

[7] B. E. Bayer. Color imaging array, July 1976. US Patent 3,971,065. 1

[8] J. Compton. Color filter array 2.0, June 2007. http://johncompton.pluggedin.kodak. com. 1

[9] P. E. Debevec and J. Malik. Recovering High Dynamic Range Radiance Maps from Photographs. In Proc. ACM Siggraph, pages 369-378, 1997. 1

[10] T. Georgiev, A. Lumsdaine, and S. Goma. High Dynamic Range Image Capture with Plenoptic 2.0 Camera. In Proc. of Signal Recovery and Synthesis, pages 1-3, 2009. 1

[11] I. Ihrke, G. Wetzstein, and W. Heidrich. A Theory of Plenoptic Multiplexing. In IEEE CVPR, 2010. oral. 1, 8

[12] D. Lanman, R. Raskar, A. Agrawal, and G. Taubin. Shield Fields: Modeling and Capturing 3D Occluders. ACM Trans. Graph. (Siggraph Asia), 27(5):131:1-131:10, 2008. 1, 2
[13] M. Levoy, R. Ng, A. Adams, M. Footer, and M. Horowitz. Light field microscopy. ACM Trans. Graph., 25(3):924-934, 2006. 1

[14] G. Lippmann. La Photographie Intégrale. Academie des Sciences, 146:446-451, 1908. 1

[15] B. Masia, S. Agustin, R. Fleming, O. Sorkine, and D. Gutierrez. Evaluation of Reverse Tone Mapping through Varying Exposure Conditions. ACM Trans. Graph. (Siggraph Asia), 28(5):1-8, 2009. 1, 8

[16] M. McGuire, W. Matusik, H. Pfister, B. Chen, J. F. Hughes, and S. K. Nayar. Optical Splitting Trees for High-Precision Monocular Imaging. IEEE Comput. Graph. Appl., 27(2):3242, 2007. 1

[17] T. Mitsunaga and S. K. Nayar. Radiometric Self Calibration. In IEEE CVPR, pages 374-380, 1999. 1

[18] S. Narasimhan and S. Nayar. Enhancing Resolution along Multiple Imaging Dimensions using Assorted Pixels. IEEE Trans. PAMI, 27(4):518-530, Apr 2005. 1

[19] S. Nayar and T. Mitsunaga. High Dynamic Range Imaging: Spatially Varying Pixel Exposures. In IEEE CVPR, volume 1, pages 472-479, Jun 2000. 1, 4, 5

[20] R. Ng, M. Levoy, M. Brodif, G. Duval, M. Horowith, and P. Hanrahan. Light Field Photography with a Hand-Held Plenoptic Camera. Technical report, Stanford CSTR 200502, 2005. 1

[21] T. Olofsson. Deconvolution and Model-Based Restoration of Clipped Ultrasonic Signals. IEEE Trans. Instrumentation and Measurements, 54(3):1235-1240, 2005. 1, 3, 4

[22] A. G. Rempel, M. Trentacoste, H. Seetzen, H. D. Young, W. Heidrich, L. Whitehead, and G. Ward. Ldr2Hdr: OnThe-Fly Reverse Tone Mapping of Legacy Video and Photographs. In ACM Trans. Graph. (Siggraph), page 39, 2007. 1,8

[23] Y. Schechner and S. Nayar. Generalized Mosaicing: High Dynamic Range in a Wide Field of View. IJCV, 53(3):245267, 2003. 1

[24] H. Seetzen, W. Heidrich, W. Stuerzlinger, G. Ward, L. Whitehead, M. Trentacoste, A. Ghosh, and A. Vorozcovs. High Dynamic Range Display Systems. ACM Trans. Graph. (Siggraph), 23(3):760-768, 2004. 1

[25] A. Veeraraghavan, R. Raskar, A. Agrawal, R. Chellappa, A. Mohan, and J. Tumblin. Non-Refractive Modulators for Encoding and Capturing Scene Appearance and Depth. In IEEE CVPR, 2008. 1, 2

[26] A. Veeraraghavan, R. Raskar, A. Agrawal, A. Mohan, and J. Tumblin. Dappled Photography: Mask Enhanced Cameras for Heterodyned Light Fields and Coded Aperture Refocussing. ACM Trans. Graph. (Siggraph), 26(3):69, 2007. 1,2

[27] S. Wang and W. Heidrich. The Design of an Inexpensive Very High Resolution Scan Camera System. Computer Graphics Forum (Eurographics), 23(10):441-450, 2004. 4

[28] X. Zhang and D. H. Brainard. Estimation of Saturated Pixel Values in Digital Color Imaging. JOSA A, 21(12):23012310, 2004. 1 